\title{
Appropriateness of unscheduled hospital admissions from care homes
}

\author{
Authors: Jennifer K Harrison, ${ }^{\mathrm{A}^{*}}$ Iona K McKay, ${ }^{\mathrm{B}^{*}}$ Patrick Grant, ${ }^{\mathrm{C}}$ Jean Hannah ${ }^{\mathrm{D}}$ and Terence ] Quinn ${ }^{\mathrm{E}}$
}

\begin{abstract}
Unscheduled hospital admissions from care homes are common and potentially avoidable but little guidance is available as to what constitutes an appropriate hospital admission. We surveyed healthcare professionals' opinions on a range of common scenarios affecting care-home residents. We developed seven clinical vignettes and an accompanying questionnaire. We used purposive sampling to obtain opinions from relevant primary care and secondary care teams. We asked assessors to comment on whether they would favour hospital admission and to justify their response using pre-selected options and/or free text. Admission to hospital was judged inappropriate in $54.6 \%$ of responses. Opinion on admission varied according to the case, with fewer than half of respondents agreeing for three of the seven cases. Recurring themes were uncertainty around services available to care homes and anticipatory care planning. The lack of consensus suggests that concepts surrounding inappropriate care-home admission are not shared by staff who provide care for this patient group.
\end{abstract}

KEYWORDS: Care home, hospitalisation, nursing homes, questionnaire

\section{Background}

A sizeable proportion of older adults in the UK resides in care homes, and absolute numbers are increasing. ${ }^{1}$ In general, care-home residents are characterised by frailty, cognitive and functional impairments and complex comorbidity. ${ }^{2,3}$ There has been a growing recognition of the need to develop an evidence base around the provision of care in this setting, ${ }^{4}$ with evidence of wide variation in healthcare service provision.,

\footnotetext{
Authors: ${ }^{\text {A }}$ clinical research fellow, Centre for Cognitive Ageing and Cognitive Epidemiology and the Alzheimer Scotland Dementia Research Centre, University of Edinburgh, Edinburgh, UK; ${ }^{B}$ Clinical services manager and registered general nurse, Thistle Healthcare, UK; C emergency medicine consultant, Emergency Department, Western Infirmary, Glasgow, UK; Dgeneral practitioner, NHS Greater Glasgow and Clyde, Glasgow, UK; ${ }^{E}$ senior clinical lecturer and honorary consultant physician, Institute of Cardiovascular and Medical Sciences, University of Glasgow, Glasgow, UK; * Joint first authors
}

The provision of unscheduled care has been specifically highlighted as an area in which traditional approaches may be ineffective. ${ }^{7}$ There is a perception that many unscheduled admissions to acute services from care homes could be avoided. ${ }^{8}$ This aligns with a general body of health-services research about ways to reduce unscheduled admissions in older adults. ${ }^{9}$ Identification of which patients do not require admission is challenging. Care-home residents are vulnerable to unanticipated changes in health, either as a result of decompensation of chronic disease or new pathologies. Decision-making processes about hospitalisation have to balance patient and carer preferences, clinical and medicolegal risk and the perception of this risk (which might be a particular issue for those with less clinical experience),${ }^{10}$ and differing models of care. ${ }^{11}$ Various reports suggest that healthcare staff do not always make the right decision, ${ }^{7}$ and there is wide variation in GPs' perceptions about the management of carehome residents in the emergency department setting. ${ }^{12}$ For some care-home residents, an admission might be avoidable; for others an admission might not be appropriate. The care needed can often be provided without hospital admission. Various papers have described acute hospital admissions from care homes in terms of system failures, ${ }^{13,14}$ inefficient use of community resources, ${ }^{15}$ or futility associated with inevitable mortality. ${ }^{16}$ This heterogeneity suggests that we have no criteria to define an inappropriate admission from care homes.

If we are to offer guidance on 'appropriate' admissions from care homes, we need to operationalise what constitutes an 'inappropriate' admission. Our aim was to assess the opinions of multidisciplinary healthcare professionals about emergency hospital admission for a range of common scenarios affecting care-home residents.

\section{Methods}

We used a questionnaire-based approach following best practice in conduct and design of clinician-based survey research. ${ }^{17}$ The vignettes (see online-only supplementary material: S1) were designed by a single author (IKM) with input from other authors. The study was done in Glasgow, UK, which has roughly 80,000 residents older than 65 years and a care-home population of around 3,000 (3.8\%). ${ }^{18}$ Within Glasgow, certain GPs offer dedicated sessions to care homes. This collective, which is called the Nursing Home Medical Practice (NHMP), covers around $85 \%$ of Glasgow care homes 
during working hours, offering proactive assessment and reactive review. The service is multidisciplinary, comprising GPs with a special interest, allied health professionals and specialist nurses with a care-home-liaison role. The NHMP is not a gatekeeper to admission from care homes, although it audits and seeks feedback on these admissions.

We collated responses from key stakeholders in primary care staff with an interest in care homes (GPs and care-home-liaison nurses) and front-line secondary care staff (physicians working in geriatric, general and emergency medicine). These groups are most likely to have direct experience of care-home residents in acute care settings. The primary care staff who participated were all involved with the NHMP. Although most NHMP GPs share their care-home duties with standard GP commitments, we recognise that their views may not be representative of all in primary care.

We designed a series of short vignettes based on common clinical scenarios. The NHMP encourages dialogue with secondary care teams, and we had access to correspondence describing cases in which a secondary care clinician felt that referral had been inappropriate. We selected cases with repeated ideas and themes to give a core set of common care-home admission scenarios to create our vignettes. The final number of vignettes was seven, which we chose to balance richness of data against respondent burden. ${ }^{19}$

We structured the questionnaire to allow for collection of quantitative and semi-qualitative data. For each vignette, assessors were asked to make a primary judgement with a structured (binary) outcome response of whether hospital admission was appropriate or not. When an admission was judged inappropriate, options were presented for which structured (multiple choice) responses were allowed. These were grouped as: care that could be provided in the care home, in the community, or as an outpatient (avoidable admission), and as admission not appropriate for the individual patient (inappropriate admission). A free-text box was included for comments to be recorded.

The questionnaire was piloted using a modified Delphi technique, with input from seven healthcare professionals. After first-round piloting, we sought information on flow, salience, acceptability and administrative ease. ${ }^{20}$ We revised the questionnaires and vignettes as an iterative process, and after two rounds of amendment all were happy with the content. We used a purposive sampling approach ${ }^{21}$ to target the groups of interest. We used snowball sampling to ensure comprehensive distribution. Questionnaires were circulated by email or in person, along with a participation information sheet, consent form and cover letter. We identified a study lead, who distributed questionnaires and encouraged completion.

We sent one reminder if no response was received. To minimise responder bias, the questionnaire replies were returned to a study email account and were anonymised by an independent person before being forwarded to the analysing researcher.

For each vignette, we described percentage of responses to pre-specified items. Our primary analysis of interest was comparison of care-home-specialist primary care staff and secondary care staff. We used standard statistical descriptors, and present most our data as n (\%). For quantitative analyses, we used the $X^{2}$ test and proportional differences, with corresponding $95 \%$ confidence intervals (CIs) and defined significance at the threshold of $\mathrm{p}<0.05$. Statistics were calculated using Minitab 16.2.1 (LEADTOOLS 1991-2004, LEAD Technologies). The free-text sections allowed for a semi-qualitative analysis; we described a thematic analysis using codes to identify common themes, and used the constant comparative approach. ${ }^{22}$ Ethical permission for the study was granted by the University Research Ethics Committee of the University of Dundee (UREC 12159).

\section{Results}

Our target population comprised 80 healthcare professionals, of whom 50 responded $(62.5 \%)$. Four questionnaires (8\%) were incomplete; we included only completely answered vignettes. Those in secondary care were more likely (38\%, 95\% CI 19-58, $\mathrm{p}<0.0001)$ to complete and return the questionnaire than those in primary care (Table 1).

Responses varied across all the vignettes presented, and not all questionnaires had a response for every vignette presented. Summating all the responses from the seven vignettes, around half felt that admission was inappropriate $(55 \% \mathrm{n}=189 / 346)$. (Table 2). Opinions about the need for admission varied according to the individual vignette and also by respondents' background.

When admission was thought inappropriate, the questionnaire offered two choices, 'appropriate care could be delivered in community or care home' or 'admission to acute hospital not in patient's best interests'. Most respondents who completed these sections chose both options together, corresponding to $85 \%$ of responses (161/189) and $88 \%$ of responses (166/189) respectively. Looking at individual vignettes, there was almost complete agreement that admission was inappropriate for vignette one (advanced dementia, which was deemed inappropriate by 47 of 50 respondents (94\%)). Most respondents felt that vignette two warranted admission (hip pain and reduced mobility, deemed inappropriate by only 14 of 50 respondents (28\%)). For most other vignettes, opinions about the appropriateness of admission were split more evenly (Table 2 ).

There was disagreement on the need for admission both between and within professional groups. Overall, GPs and care-home-liasion nurses were significantly more likely to think admission to hospital was inappropriate than were colleagues in secondary care (proportional difference 13\%, 95\% CI 2-24, $\mathrm{p}=0.017$ ). The sample sizes of individual disciplines within secondary care were too small to allow meaningful analyses of between-group differences.

\section{Table 1. Return rate of questionnaires by professional group}

$\begin{array}{ll}\text { Professional group } & \mathbf{n} / \mathbf{N}(\%) \\ \text { Specialist general practitioner } & 10 / 28(35.7) \\ \text { Care-home-liaison nurse } & 8 / 13(61.5) \\ \text { Geriatric medicine physician } & 11 / 15(73.3) \\ \text { General medicine physician } & 7 / 10(70.0) \\ \text { Emergency medicine physician } & 14 / 14(100.0) \\ \text { Total } & 50 / 80(62.5)\end{array}$


Table 2. Summary of responses by vignette.

Vignette

1: advanced dementia with behavioural disturbance

Overall

Primary care

Secondary care

2: reduced mobility and hip pain in a patient with dementia

Overall

Primary care

Secondary care

3: dementia with loss of safe swallow

Overall

Primary care

Secondary care

4: exacerbation of chronic condition (chronic obstructive pulmonary disease)

Overall

Primary care

Secondary care

5: acute problem requiring procedural skill (urinary retention)

Overall

Primary care

Secondary care

6: exacerbation of chronic condition (single self-terminating seizure in patient with epilepsy)

Overall

Primary care

Secondary care

7: probable iatrogenic harm (minor haematemesis in patient with polypharmacy)

Overall

Primary care

Secondary care

\section{All combined}

Overall

Primary care

Secondary care
Admission

deemed

inappropriate,

$n / N(\%)$

$47 / 50(94)$
$18 / 18(100)$
$29 / 32(91)$

$14 / 50$ (28)

$5 / 18(28)$

9/32 (28)

$34 / 50$ (68)

16/18 (89)

18/32 (56)

20/50 (40)

$9 / 18(50)$

$11 / 32(34)$

26/50 (52)

$10 / 18(56)$

16/32 (50)

25/48 (52)

10/18 (56)

$15 / 30(50)$

$21 / 48(44)$

10/17 (59)

$11 / 29$ (38)
Problem could be managed in care-

home or as an

outpatient, $n / \mathrm{N}$ (\%)

44/47 (94)

-

$-$

$9 / 14(64)$

$-$

$-$

29/34 (85)

$-$

-

$18 / 20(90)$

-

$24 / 26(92)$

$-$

$-$

$21 / 25(84)$

$-$

$-$

16/21(76)

-

-

$161 / 189$ (85)

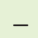

$78 / 125$ (62)

$109 / 221$ (49)

47/47 (100)

$9 / 14(64)$

$32 / 34(94)$

$17 / 20(85)$

$21 / 26(81)$

20/21 (95)

166/189 (88)

Admission not ate fo the individual patient, n/N (\%)

$-$

$-$

$20 / 25(80)$

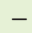

$-$

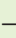

$-$

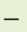

$-$

Data are $\mathrm{n} / \mathrm{N}(\%)$. Primary care includes care-home-liaison nurses and specialist GPs; secondary care includes emergency medicine specialists, geriatricians and general medical physicians. In columns three and four, only responses by those who deemed the admission inappropriate are included.

When we review the free-text responses, common themes emerged for each vignette and across the responses as a whole (Box 1). For vignette one, comments recognised an end-of-life situation and encouraged the use of an advance-care plan. For vignette two, common concerns expressed were around the need for radiological investigation to exclude a fracture, with respondents unclear as to how this could be achieved without referral for admission. In vignette three, respondents expressed 
Jennifer K Harrison, Iona K McKay, Patrick Grant, Jean Hannah and Terence J Quinn

Box 1. Themes emerging from responses to vignettes.

\author{
Vignette 1 theme: recognising end of life in care homes \\ 'Treatment should be palliative. Her daughter will need education and reassurance' (EM7) \\ 'This patient needs an anticipatory care plan drawn up' (GenMed5)
}

Vignette 2 theme: limiting time in hospital for essential investigations only

'Not necessarily requiring hospital admission but warrants A\&E r/v with $x$-rays to exclude \#NOF' (A\&E4)

'If possible the ideal would be assessment with $\mathrm{x}$-ray in $\mathrm{A} \& \mathrm{E}$ (phoning ahead to alert the A\&E team) and prompt return to care home if no bony injury' (Ger7)

Vignette 3 theme: difficulties of providing parenteral hydration in care homes

'May benefit from IV fluids... Can this be done at home?' (EM8)

'Short-term use of subcutaneous fluids' (CHLN6)

Vignette 4 theme: difficulties of providing parenteral treatment in care home

'The only treatment hospital can add is IV antibiotics' (Ger1)

'Would be good if IV antibiotics could be given in the care home setting if required' (CHLN8)

Vignette $\mathbf{5}$ theme: difficulties around providing medical procedures in care homes

'Needs catheter change for urinary retention. Would be nice if this were achievable in the nursing home with outreach services, but my experience is these patients often end up in A\&E' (EM2)

'This gentleman requires a change of catheter with gentamicin cover' (GenMed4)

Vignette 6 theme: accessing specialist review in care homes

'Could patient be discussed with epilepsy team rather than directly admitted?' (Ger4)

'follow up with neurology outreach services?' (CHLN8)

\title{
Vignette 7 theme: managing complexity in care homes
}

'She needs bloods including INR [international normalised ratio] \& urea \& haemoglobin. She needs a medication review' (EM7)

'Could be managed in care home .... but would take a lot of organising' (Ger7)

\section{Themes common to various scenarios}

Using advance-care planning (ACP): 'although prognosis is guarded, frailty does not equal do not treat actively - there really needs to be discussion re: ACP' (GenMed5), 'ACP would be appropriate with GP and multidisciplinary team' (CHLN3)

Communication between the patient, their family, care home staff and the healthcare team: 'The patient's care would be discussed using a multidisciplinary approach' (CHLN7)

a lack of awareness of the potential treatment available within the care-home setting. In both vignettes three and four, there was a similar lack of consensus over the route of administration of antibiotics, with those favouring intravenous administration suggesting admission was necessary. Respondents also noted the potential role for advance-care planning in view of the likelihood of recurrence. In vignette five, respondents expressed a lack of awareness of the facilities available in the community to manage urinary catheterisation, often indicating that such a procedure would necessitate admission to allow for intravenous antibiotic cover. Similarly in vignette six, respondents cited the need for specialist epilepsy management, which they felt could be accessed via hospital admission only. Finally, the need for the patient in vignette seven to have blood tests and a medication review was given as the reason to arrange admission to hospital. Respondents expressed concern about mechanisms for followup in the community if admission were not arranged.

\section{Discussion}

In this questionnaire-based study, we found that there was a lack of consensus among clinicians as to when a care-home resident should be referred to hospital, with primary care teams more likely to recommend treating patients in the care home. When admission was not recommended, responses suggested that the rationale for this decision was based partly on 'appropriateness' of admission of frail elderly patients and partly on the clinical indication for secondary care and whether admission was 'avoidable'. Common themes that emerged from free-text responses related to communication, recognition of end of life, advance-care planning and access to specialist review and/or interventions in the care-home setting. The lack of consensus around suitability for hospital admission suggests that concepts of 'inappropriate care-home admission' are not shared by the various staff groups who provide usual care for these patients. We cannot comment on whether primary or secondary care respondents made the correct choice around management, although all the vignettes are based on original patient cases in which it was felt that admission had not been in the patient's best interests. The overall trend was that secondary care clinicians were more likely to favour admission of care-home residents. In the context of heated debate around the gatekeeping role of primary care, our data suggest that primary care doctors with a special 
interest and care-home-liaison nurses might be less risk averse and more comfortable managing complexity in the community than colleagues in secondary care realise.

A recurring theme in free-text responses concerned availability of services, expertise and interventions in the carehome setting. This finding is in keeping with results from other healthcare systems. ${ }^{23,24}$ Responses suggest a lack of awareness of the services available, and addressing these knowledge gaps must be a priority if we are to improve care delivered to this vulnerable patient group. Comments about availability of specialist opinion or immediate access to investigations to prevent admission are a concern, and suggest that the interface between care homes and secondary care could be improved. There are examples of successful projects that can safely keep medically unwell care-home residents in their preferred environment. Various groups have created clinical pathways for the management of pneumonia in care homes, ${ }^{25}$ and have used improved access to geriatrician advice to reduce hospital admissions. $^{26}$

When admission was not recommended, we offered respondents choices of 'avoidable' or 'inappropriate' admission distinct categories that clearly often coexist because most respondents selected both options. Gott and colleagues assessed the appropriateness of admissions for adults with palliativecare needs and reported that, although most admissions were deemed appropriate, the inappropriate group comprised predominantly older adults living in residential or nursing care. ${ }^{27}$ For avoidable admissions, previous research suggested that, although $55 \%$ of emergency department attendances from care homes were potentially preventable, most of these patients were still admitted to hospital, ${ }^{14}$ showing that a theoretically preventable admission is not the same as a patient who can be discharged. Once a care-home resident arrives in a busy emergency department, it can be quite challenging to assess fully, treat, and discharge them within current recommended time frames (ie 4 hours). Formal admission to an inpatient ward might often be seen as the path of least resistance, even if it is not always necessary from a medical point of view.

A potentially helpful approach is the use of screening tools in the emergency department which have been developed to identify older adults who are at high risk of adverse outcomes targeting them for comprehensive geriatric assessment interventions. ${ }^{28}$ Delivering comprehensive geriatric assessments to frail older people within the emergency department in a frailty unit reduced admission to hospital without evidence of early readmission. ${ }^{29}$ Although not specifically designed for carehome residents, such approaches have the potential to support the assessment of, and decision making for, complex frail older adults such as care-home residents. Alternative approaches include specialist in-reach teams to support care-home staff, which have been associated with reductions in admission to hospital through provision of care at the care home. ${ }^{30}$

None of our case vignettes included the use of advancecare planning, reflecting the cases they were derived from. However, targeted advance-care-planning interventions reduce admissions to hospital among care-home residents and were associated with reductions in mortality in one study. ${ }^{31}$ Improving end-of-life care for care-home residents is also reliant on the establishment of effective working relationships with primary care to support care-home staff. ${ }^{32}$

\section{Box 2. Questions for future research.}

What are the views and experiences of care home residents on hospital admissions and their perception of appropriateness?

What are the common precipitants of unscheduled admission from care homes and what are the outcomes?

$>$ Would educating non-specialist primary care and secondary care staff around community services affect admissions from care homes?

Can we design and trial services that provide high-quality specialist care to care-home residents who become unwell?

The strength of our approach was the use of real-world cases to assess opinions on admission. The response rate was good for this type of survey, and we were particularly encouraged by the $100 \%$ response from emergency department clinicians. The engagement with the study suggests that stakeholders all recognise the difficulty of managing this patient group. We recognise limitations in our approach. Our sample size is modest and not powered to analyse results by individual specialty groups, and any questionnaire-based survey can be prone to responder biases. The GP and nurse respondents were clinicians with an interest in care-home-based healthcare and results might have been different had we targeted nonNHMP staff. We also acknowledge that our work did not seek the opinions of patients about their experiences of hospital admission and their perspectives regarding appropriateness. Although beyond the scope of our project, this is an important area, and further research is needed. Additional research questions arising from this work are posed in Box 2.

\section{Conclusion}

Our research demonstrates that there is a lack of consensus about what constitutes an inappropriate admission. The variation in proposed treatments offered for our vignettes highlights the difficulty in managing care-home residents. There is a degree of subjectivity, and decisions need to be made on a case-by-case basis. As regards appropriateness of admission, we are probably no closer to offering universal guidance and a consensus across the various disciplines seems unlikely. Many responders felt that certain admissions could have been avoided. Improving knowledge, awareness and access to community services should allow for the delivery of improved healthcare for care-home residents, without denying access to necessary interventions and support.

Mapping secondary care staff's knowledge about community services available to care homes might help with the targeting of education and awareness. A model of care with greater access to secondary care expertise and interventions (shortterm parenteral therapy, for example) could plausibly have an impact on admissions but, ideally, would need to be assessed in a clinical study before implementation.

\section{Acknowledgements}

We would like to thank everyone who participated in the study through questionnaire completion. 


\section{Funding}

TJQ is supported by a joint Stroke Association/Chief Scientist Office Senior Clinical Lectureship. JKH is supported by a clinical research fellowship from Alzheimer Scotland and the University of Edinburgh Centre for Cognitive Ageing and Cognitive Epidemiology, part of the cross council Lifelong Health and Wellbeing Initiative (MR/L501530/1). Funding from the Biotechnology and Biological Sciences Research Council and Medical Research Council is gratefully acknowledged. The funders played no part in the design, analysis or conduct of the study.

\section{References}

1 Audit Scotland. Reshaping care for older people. Edinburgh: Audit Scotland, 2014.

2 Gordon A, Franklin M, Bradshaw L et al. Health status of UK care home residents: a cohort study. Age Ageing 2014;43:97-103.

3 Bowman C, Whistler J, Ellerby M. A national census of care home residents. Age Ageing 2004;33:561-6.

4 Burns E, Nair S. New horizons in care home medicine. Age Ageing 2014;43:2-7.

5 British Geriatrics Society. Quest for quality. An Inquiry into the quality of healthcare support for older people in care homes: a call for leadership, partnership and improvement. London: British Geriatrics Society, 2011.

6 British Geriatrics Society. Failing the frail: a chaotic approach to commissioning healthcare services for care homes. London: British Geriatrics Society, 2010.

7 Smith P, Sherlaw-Johnson C, Ariti C, Bardsley M. Quality Watch. Focus on: Hospital admissions from care homes. London: Health Foundation/Nuffield Trust, 2015.

8 Bowman C, Elford J, Dovey J, Campbell S, Barrowclough H. Acute hospital admissions from nursing homes: some may be avoidable. Postgrad Med J 2001;77:40-2.

9 Imison C, Poteliakhoff E, Thompson J. Older people and emergency bed use - exploring variations. London: The King's Fund, 2012.

10 McDermott C, Copping R, Little P, Leydon G. Hospital admissions from nursing homes: a qualitative study of GP decision making. $\mathrm{Br}$ J Gen Pract 2012;62:e538-45.

11 Donald I, Gladman J, Conroy S et al. Care home medicine in the UK - in from the cold. Age Ageing 2008;37:618-20.

12 Carter L, Skinner J, Robinson S. Patients from care homes who attend the emergency department: could they be managed differently. Emerg Med J 2009;26:259-62.

13 Godden S, Pollock A. The use of acute hospital services by elderly residents of nursing and residential care homes. Health Soc Care Comm 2001;9:367-74.

14 Briggs R, Coughlan T, Collins R, O'Neill D, Kennelly S. Nursing home residents attending the emergency department: clinical characteristics and outcomes. QJM 2013;106:803-8.

15 Quinn T. Emergency hospital admissions from care-homes: who, why and what happens? A cross-sectional study. Gerontology 2011;57:115-20.

16 Alrawi Y, Parker R, Harvey R et al. Predictors of early mortality among hospitalized nursing home residents. QJM 2013;106:51-7.
17 Burns K, Duffett M, Know M et al. A guide for the design and conduct of self-administered surveys of clinicians. CMAJ 2008; 179:245-52.

18 Care home framework tender, 2014. www.glasgow.gov.uk/ CHttpHandler.ashx?id=18980\&p=0 [Accessed 22 July, 2015].

19 Fox J. Designing research: basics of survey construction. Minim Invasive Surg Nurs 1994;8:77-9.

20 Bowden A, Fox-Rushby J, Nyandieka L, Wanjau J. Methods for pre-testing and piloting survey questions: illustrations from the KENQOL survey of health-related quality of life. Health Pol Planning 2002;17:322-30.

21 Green J, Thorogood N. Qualitative methods for health research, 2nd edn. London: Sage Publications, 2009.

22 Barbour R. Introducing qualitative research - a student guide to the craft of doing qualitative research. London: Sage Publications, 2008.

23 Ouslander J, Lamb G, Perloe M et al. Potentially avoidable hospitalizations of nursing home residents: frequency, causes, and costs. J Am Geriatr Soc 2010;58:627-35.

24 Ong A, Sabanathan K, Potter J, Myint P. High mortality of older patients admitted to hospital from care homes and insight into potential interventions to reduce hospital admissions from care homes: the Norfolk experience. Arch Gerontol Geriatr 2011;53:316-9.

25 Loeb M, Carusone S, Goeree R et al. Effect of a clinical pathway to reduce hospitilizations in nursing home residents with pneumonia: a randomized controlled trial. JAMA 2006;295:2503-10.

26 Lisk R, Yeong K, Nasim A et al. Geriatrician input into nursing homes reduces emergency hospital admissions. Arch Gerontol Geriatr 2012;55:331-7.

27 Gott M, Gardiner C, Ingleton C et al. What is the extent of potentially avoidable admissions among hospital inpatients with palliative care needs? BMC Palliat Care 2013;12:9.

28 Graf C, Zekry D, Giannelli S, Michel J, Chevalley T. Efficiency and applicability of comprehensive geriatric assessment in the emergency department: a systematic review. Aging Clin Exper Res 2011;23:244-54.

29 Conroy S, Ansari K, Williams M et al. A controlled evaluation of comprehensive geriatric assessment in the emergency department: the 'emergency frailty unit'. Age Ageing 2014;43:109-14.

30 Szczepura A, Nelson S, Wild D. In-reach specialist nursing teams for residential care homes: uptake of services, impact on care provision and cost-effectiveness. BMC Health Serv Res 2008;8:269.

31 Caplan G, Meller A, Squires B, Chan S, Willett W. Advance care planning and hospital in the nursing home. Age Ageing 2006;35:581-5.

32 Seymour JE, Kumar A, Froggatt K. Do nursing homes for older people have the support they need to provide end-of-life care? A mixed methods enquiry in England. Palliat Med 2011;25:125-38.

Address for correspondence: Dr JK Harrison, Geriatric Medicine, Room S1642, Royal Infirmary of Edinburgh, 51 Little France Crescent, Edinburgh EH16 4SB, UK.

Email: jenni.harrison@ed.ac.uk 